\title{
Retrospective analysis of pacritinib in patients with myelofibrosis and severe thrombocytopenia
}

\author{
Srdan Verstovsek, ${ }^{1}$ Ruben Mesa, ${ }^{2}$ Moshe Talpaz, ${ }^{3}$ Jean-Jacques Kiladjian, ${ }^{4}$ Claire N. Harrison, ${ }^{5}$ \\ Stephen T. Oh, ${ }^{6}$ Alessandro M. Vannucchi, ${ }^{7}$ Raajit Rampal, ${ }^{8}$ Bart L. Scott, ${ }^{9}$ Sarah A. Buckley, ${ }^{10}$ \\ Adam R. Craig, ${ }^{10}$ Karisse Roman-Torres ${ }^{10}$ and John O. Mascarenhas ${ }^{11}$

\begin{abstract}
${ }^{1} \mathrm{MD}$ Anderson Cancer Center, Houston, TX, USA; ${ }^{2}$ Mayo Clinic, Scottsdale, AZ, USA; ${ }^{3}$ University of Michigan, Comprehensive Cancer Center, Ann Arbor, MI, USA; ${ }^{4}$ Hôpital SaintLouis, Université Paris Diderot, Paris, France; ${ }^{5}$ Guy's and St Thomas' NHS Foundation Trust, London, UK; ${ }^{6}$ Washington University School of Medicine, St. Louis, MO, USA; ${ }^{7}$ University of Florence, Azienda Ospedaliera-Universitaria Careggi, Florence, Italy; ${ }^{8}$ Memorial Sloan Kettering Cancer Center, New York, NY, USA; ${ }^{9}$ Fred Hutchinson Cancer Research Center, Seattle, WA, USA; ${ }^{10} \mathrm{CTI}$ BioPharma Inc, Seattle, WA, USA and ${ }^{11} \mathrm{Icahn}$ School of Medicine at Mount Sinai, New York, NY, USA
\end{abstract}

\author{
Correspondence: \\ Srdan Verstovsek \\ sverstov@mdanderson.org \\ Received: June 28, 2021. \\ Accepted: September 15, 2021 \\ Prepublished: September 23, 2021. \\ https://doi.org/10.3324/haematol.2021.279415 \\ @2022 Ferrata Storti Foundation \\ Haematologica material is published under \\ a CC-BY-NC license (c) (1) $\$$
}

\begin{abstract}
Thrombocytopenia is common in patients with myelofibrosis (MF) and is a well-established adverse prognostic factor. Both of the approved Janus kinase (JAK) inhibitors, ruxolitinib and fedratinib, can worsen thrombocytopenia and have not been evaluated in patients with severe thrombocytopenia (platelet counts $<50 \times 10 \% / L$ ). Pacritinib, a novel JAK2/interleukin-1 receptor-associated kinase 1 inhibitor, has been studied in two phase III trials (PERSIST-1 and PERSIST2), both of which enrolled patients with MF and severe thrombocytopenia. In order to better characterize treatment outcomes for this population with advanced disease, we present a retrospective analysis of efficacy and safety data in the 189 patients with severe thrombocytopenia treated in the PERSIST studies. The proportion of patients in the pacritinib group meeting efficacy endpoints was greater than in the BAT group for $\geq 35 \%$ spleen volume reduction $(23 \%$ vs. $2 \%$, $P=0.0007), \geq 50 \%$ modified Total Symptom Score reduction (25\% vs. $8 \%, P=0.044$ ), and self-reported symptom benefit ("much" or "very much" improved; $25 \%$ vs. $8 \%, P=0.016$ ) at the primary analysis time point (week 24). The adverse event profile of pacritinib was manageable, and dose modification was rarely required. There was no excess in bleeding or death in pacritinib-treated patients. These results indicate that pacritinib is a promising treatment for patients with MF who lack safe and effective therapeutic options due to severe thrombocytopenia.
\end{abstract}

\section{Introduction}

Patients with myelofibrosis (MF) who have severe thrombocytopenia (platelet counts $<50 \times 10^{\%} / \mathrm{L}$ ) comprise a subset of patients with cytopenic MF who generally have more advanced disease, including anemia, greater risk of bleeding, worse symptom burden, higher risk of leukemic transformation, and shorter survival (median 15 months) compared with patients with higher platelet counts. ${ }^{1-3}$ These patients lack effective treatment options and are often excluded from clinical trials. Neither ruxolitinib nor fedratinib, the only drugs currently approved for MF, has been studied in patients presenting with severe thrombocytopenia, and neither drug has a product label with a recommended starting dose for this population..$^{4-9}$ Furthermore, both have been shown to cause treatment-related thrombocytopenia, which requires dose modification and may result in reduced efficacy. For example, patients treated with ruxolitinib at $\leq 10 \mathrm{mg}$ twice a day (BID) were less likely to achieve significant spleen volume responses. ${ }^{10}$ Development of cytopenias was the most common reason for patients discontinuing ruxolitinib. ${ }^{11}$ Ruxolitinib has been tested in patients with moderate thrombocytopenia (platelet counts $\left.50-100 \times 10^{\%} / L\right)$. In the phase Ib EXPAND study, dose interruptions or reductions were required in $89 \%$ of patients who had platelet counts $50-74 \times 10^{\%} / \mathrm{L}$, and $78 \%$ experienced grade 3 or 4 thrombocytopenia as an adverse event..$^{12}$ In the phase IIIb expanded-access JUMP study, $55 \%$ of patients who started on ruxolitinib $5 \mathrm{mg}$ BID required further dose reduction, and grade 3 or 4 thrombocytopenia was a common adverse event. ${ }^{13}$ There is a significant unmet need for effective and safe therapies for patients living with MF and experiencing severe thrombocytopenia, who may comprise up to $35 \%$ of the MF population. ${ }^{14}$

Pacritinib is a novel inhibitor of Janus kinase 2 (JAK2) and interleukin-1 receptor-associated kinase $1^{15}$ currently in development for patients with MF and thrombocytopenia. Two randomized controlled phase III trials, PERSIST- $1^{16}$ and 
PERSIST-2, ${ }^{17}$ have compared the efficacy and safety of pacritinib versus best available therapy (BAT). Because earlier phase I-II studies had shown that pacritinib has limited myelosuppressive properties, ${ }^{18}$ neither phase III study had a lower limit on platelet counts, and both included patients with moderate and severe thrombocytopenia. The PERSIST studies represent the largest published data set of patients with MF and severe thrombocytopenia treated in randomized controlled trials. In order to better characterize treatment outcomes for this population with advanced disease, we present pooled data from patients with severe thrombocytopenia treated in PERSIST-1 and PERSIST-2 and describe the efficacy and safety profiles of pacritinib compared to BAT.

\section{Methods}

The PERSIST-1 (clinicaltrials gov. Identifier: NCT01773187) and PERSIST-2 (clinicaltrials gov. Identifier: NCT02055781) study designs and methodology have been previously described. ${ }^{16,17}$ Key features of both studies are summarized below. The study protocols were approved by the Institutional Review Boards at each study site and the study was conducted in accordance with the Declaration of Helsinki. All patients provided written informed consent. Both studies included adult patients with either primary or secondary MF. Patients had intermediate-1, intermediate-2, or high-risk disease, as categorized by the Dynamic International Prognostic Scoring System, and palpable splenomegaly $\geq 5 \mathrm{~cm}$ below the left costal margin. PERSIST-1 enrolled patients regardless of platelet count, while PERSIST-2 was restricted to patients with platelet counts $\leq 100 \times 10^{9} / \mathrm{L}$. Both studies included patients with severe thrombocytopenia (platelet counts $<50 \times 10^{9} / \mathrm{L}$ ) at baseline; this population comprised $16 \%$ of patients in PERSIST- 1 and $45 \%$ in PERSIST-2. Prior use of JAK inhibitors was permitted only in PERSIST-2. PERSIST-1 randomized patients 2:1 to receive pacritinib $400 \mathrm{mg}$ daily or BAT. PERSIST-2 randomized patients 1:1:1 to receive pacritinib $400 \mathrm{mg}$ daily, pacritinib $200 \mathrm{mg}$ BID, or BAT. Randomization was stratified by baseline platelet count. BAT included any available physicianselected treatment, including "watch and wait" (i.e., no active treatment). Ruxolitinib was included as an option only in PERSIST-2. Fedratinib was not available as BAT in either study. Patients randomized to receive BAT were allowed to cross over to pacritinib at 24 weeks or at disease progression. Safety and efficacy data were censored at the time of crossover.

\section{Statistical analysis}

Patients in PERSIST- 1 and PERSIST-2 with baseline platelet counts $<50 \times 10^{9} / \mathrm{L}$ were included in the analysis. Efficacy endpoints were assessed at week 24 and included the percentage of patients achieving $\geq 35 \%$ spleen volume response (SVR), the percentage achieving $\geq 50 \%$ reduction in the modified Total Symptom Score (TSS) v2.0, ${ }^{19}$ and the percentage reporting symptoms as "much" or "very much" improved on the Patient Global Impression of Change scale. Cardiac and hemorrhagic events were defined using Standardized MedDRA Queries. Since the PERSIST-2 study was terminated prematurely due to a clinical hold, intention-to-treat (ITT) efficacy analyses included all randomized patients in PERSIST- 1 and the $71 \%$ in PERSIST- 2 who were randomized at least 22 weeks prior to the hold. As the TSS instrument administered during PERSIST-1 was changed from v1.0 to v2.0 part-way through the study, only patients who had completed v2.0 at baseline were included in the ITT TSS analysis. Safety analyses included all treated patients (Online Supplementary Figure S1). Differences in baseline characteristics between groups were evaluated using the chi-square test (categorical variable) or the Wilcoxon rank-sum test (continuous variables). The Breslow and Day homogeneity test was performed to measure the magnitude of treatment effect on the response in the presence of a prior JAK or MF diagnosis. For efficacy outcomes, Fisher's exact test was used to perform between-group categorical analysis; the Wilcoxon exact test was used for continuous outcome variables.

\section{Results}

\section{Patient characteristics}

In total, 192 patients (133 pacritinib, 59 BAT) with severe thrombocytopenia were enrolled in PERSIST-1 and PERSIST-2. Of these, 189 (132 pacritinib, 57 BAT) received at least one dose of study drug. There were 152 patients (104 pacritinib, 48 BAT) included in the ITT efficacy population, and 117 patients (80 pacritinib, 37 BAT) completed the TSS v2.0 at baseline (Online Supplementary Figure S1). As shown in Table 1, median age was 69 years (range, 50-91). The majority of patients (72\%) had primary MF, with a median time from diagnosis of 2.0 years. Approximately one-third of patients (34\%) had received prior treatment with a JAK2 inhibitor. Median platelet count at baseline was $28 \times 10 \% / \mathrm{L}, 63.5 \%$ had hemoglobin $<10 \mathrm{~g} / \mathrm{dL}$, and $48 \%$ had $\geq 1 \%$ peripheral blood blasts. Approximately half of the patients (49\%) had grade 3 marrow fibrosis, and $38 \%$ had low or normal marrow cellularity ( $\leq 40 \%)$. The most common therapies selected as BAT were "watch and wait" (37\%; with $25 \%$ receiving only "watch and wait" for the duration of the study), ruxolitinib (30\%; only available for PERSIST-2), hydroxyurea (28\%), and prednisone (12\%). The duration of study drug exposure was similar for pacritinib and BAT (median 5.5 and 5.2 months, respectively), although $33 \%$ of patients on BAT cycled through multiple therapies on study. Shorter treatment durations were due, 
in part, to truncation at the time of the clinical hold, as $46 \%$ of patients were still on pacritinib at the time of the clinical hold. The median total daily dose of pacritinib re- mained $400 \mathrm{mg}$ at both weeks 12 and 24, whereas patients in PERSIST-2 who received ruxolitinib as BAT were prescribed a median post-titration dose of $10 \mathrm{mg}$ and were

Table 1. Baseline patient and disease characteristics in patients treated with pacritinib or best available therapy

\begin{tabular}{|c|c|c|c|}
\hline & Pacritinib $(\mathbf{N}=132)$ & BAT $(\mathbf{N}=57)$ & P-value** \\
\hline Age, median (range) & $69(50-91)$ & $69(50-84)$ & 0.95 \\
\hline Male sex, N (\%) & $80(61)$ & $28(49)$ & 0.14 \\
\hline $\begin{array}{l}\text { ECOG PS, } N^{*}(\%) \\
0-1 \\
2-3\end{array}$ & $\begin{array}{l}100 / 132(76) \\
32 / 132(24)\end{array}$ & $\begin{array}{l}42 / 55(76) \\
13 / 55(24)\end{array}$ & 0.93 \\
\hline Prior JAK2 inhibitor, N (\%) & $43(33)$ & $21(37)$ & 0.57 \\
\hline $\begin{array}{l}\text { MF diagnosis, N (\%) } \\
\text { Primary MF } \\
\text { PPV-MF } \\
\text { PET-MF }\end{array}$ & $\begin{array}{l}98(74) \\
20(15) \\
14(11)\end{array}$ & $\begin{array}{l}38(67) \\
8(14) \\
22(39)\end{array}$ & 0.17 \\
\hline Time since MF diagnosis (years), median (IQR) & $2.0(0-27)$ & $2.6(0-14)$ & 0.72 \\
\hline $\begin{array}{l}\text { DIPSS risk category, N (\%) } \\
\text { Intermediate-1 } \\
\text { Intermediate-2 } \\
\text { High }\end{array}$ & $\begin{array}{l}26(20) \\
63(48) \\
43(33)\end{array}$ & $\begin{array}{c}5(9) \\
30(53) \\
22(39)\end{array}$ & 0.17 \\
\hline $\begin{array}{l}\text { Reticulin and collagen fibrosis staging, } \mathrm{N}^{*}(\%) \\
\text { MF } 0-1 \\
\text { MF } 2 \\
\text { MF } 3\end{array}$ & $\begin{array}{l}18 / 122(15) \\
38 / 122(31) \\
66 / 122(54)\end{array}$ & $\begin{array}{l}11 / 52(21) \\
15 / 52(29) \\
26 / 52(50)\end{array}$ & 0.58 \\
\hline $\begin{array}{l}\text { Bone marrow cellularity, } \mathrm{N}^{*}(\%) \\
<20 \% \\
20-40 \% \\
41-100 \%\end{array}$ & $\begin{array}{l}27 / 110(25) \\
18 / 110(16) \\
65 / 110(59)\end{array}$ & $\begin{array}{c}18 / 49(37) \\
8 / 49(16) \\
23 / 49(47)\end{array}$ & 0.26 \\
\hline $\begin{array}{l}\text { Bone marrow blast category, } \mathrm{N}^{*}(\%) \\
\geq 1 \% \\
<1 \%\end{array}$ & $\begin{array}{l}96 / 115(84) \\
19 / 115(17)\end{array}$ & $\begin{array}{l}41 / 50(82) \\
11 / 50(18)\end{array}$ & 0.82 \\
\hline $\begin{array}{l}\text { Peripheral blood blasts category, } \mathrm{N}^{*}(\%) \\
\geq 1 \% \\
<1 \%\end{array}$ & $\begin{array}{l}60 / 118(51) \\
58 / 118(49)\end{array}$ & $\begin{array}{l}31 / 50(62) \\
19 / 50(38)\end{array}$ & 0.18 \\
\hline Platelet count (10\%/L), median (range) & $29(6-49)$ & $25(5-49)$ & 0.27 \\
\hline Hemoglobin <10 g/dL, N* (\%) & $85 / 132(64)$ & $35 / 56(63)$ & 0.80 \\
\hline $\begin{array}{l}\text { RBC transfusion dependence }{ }^{\dagger}, \mathrm{N}(\%) \\
\text { Dependent } \\
\text { Independent } \\
\text { Indeterminate }\end{array}$ & $\begin{array}{l}38(29) \\
61(46) \\
33(25)\end{array}$ & $\begin{array}{l}20(35) \\
23(40) \\
14(25)\end{array}$ & 0.66 \\
\hline Spleen volume at baseline $\left(\mathrm{cm}^{3}\right)^{\ddagger}$, median (IQR) & $2,566(1,633-3,680)$ & $2,466(1,786-3,727)$ & 0.87 \\
\hline Modified TSS score at baseline ${ }^{\ddagger}$, median (IQR) & $17(12-29)$ & $7(12-27)$ & 0.94 \\
\hline $\begin{array}{l}\text { Study enrollment, N (\%) } \\
\text { PERSIST-1 } \\
\text { PERSIST-2 }\end{array}$ & $\begin{array}{l}35(27) \\
97(73)\end{array}$ & $\begin{array}{l}15(26) \\
42(74)\end{array}$ & 0.98 \\
\hline
\end{tabular}

BAT: best available therapy; DIPSS: Dynamic International Prognostic Scoring System; ECOG PS: Eastern Cooperative Oncology Group performance status; IQR: interquartile range; JAK2: Janus kinase 2; MF: myelofibrosis; PET: post-essential thrombocythemia; PPV: post-polycythemia vera; RBC: red blood cell; TSS: Total Symptom Score. *Denominators represent non-missing values. ${ }^{* *}$ Chi-square test for categorical variables and Wilcoxon rank-sum test for continuous variables. †Per Gale criteria. ${ }^{24} \ddagger$ Baseline values reported for efficacy population. 
on this treatment for a median duration of only 3.45 months.

\section{Efficacy}

The percentage of patients treated with pacritinib who achieved a 24 -week $\geq 35 \%$ SVR response was greater than the percentage of patients treated with BAT $(23.1 \%$ vs. $2.1 \%$, $P=0.0007$ ) (Figures 1 and $2 A$ ). Higher response rates were observed in patients treated with pacritinib $200 \mathrm{mg}$ BID compared with those on $400 \mathrm{mg}$ daily (29.0\% vs. 20.5\%). The median percentage change in spleen volume was greater for pacritinib-treated patients than for BAT-treated patients $(-29.4 \%$ vs. $-1.3 \%, P<0.0001)$, and the percentage of patients who experienced any improvement $(>0 \%)$ in SVR was higher in the pacritinib compared with the BAT group $(56.7 \%$ vs. $33.3 \%, P=0.0089)$. Similarly, pacritinibtreated patients were more likely to achieve $\geq 50 \%$ modified TSS reduction at week 24 compared to BAT-treated patients (25\% vs. 8.1\%, $P=0.0441$ ) (Figures 1 and $2 B$ ), and the median reduction in modified TSS score was greater with pacritinib than with BAT $(-30.3 \%$ vs $0 \%, P=0.0036)$. Response rates for TSS were similar between the two pacritinib doses. The percentage of patients who experienced any improvement ( $>0 \%)$ in TSS was higher in the pacritinib compared to the BAT group (53\% vs. $32 \%$, $P=0.049$ ). Pacritinib-treated patients were significantly more likely than patients treated with BAT to report that their symptoms were "very much" or "much" improved at week 24 (25.0\% vs. 8.3\%, respectively; $P=0.016$ ) (Figure 3). Subgroup analyses showed that pacritinib was associated with higher response rates regardless of whether patients had received prior treatment with JAK2 inhibitors or whether they had primary versus secondary MF (Table 2).

\section{Safety}

The treatment-emergent adverse events (TEAE) that developed in patients with severe thrombocytopenia were consistent with the overall PERSIST study results and were generally grade 1 or 2 in severity (Table 3 ). The most common non-hematologic TEAE in pacritinib-treated patients were diarrhea (60.6\%; 5.3\% grade $3-4$ ), nausea (30.3\%; $1.5 \%$ grade $3-4)$, and vomiting (26.5\%; $0.8 \%$ grade $3-4)$. These gastrointestinal events were observed more frequently in the pacritinib group but rarely led to dose reduction $(3.0 \%$ for diarrhea, $1.5 \%$ for nausea) or discontinuation (3.8\% for diarrhea, $0 \%$ for nausea). The most common hematologic TEAE in this thrombocytopenic population among pacritinib-treated patients were thrombocytopenia (34.8\%) and anemia (31.8\%). Hematologic TEAE were generally grade 3 or 4 given the degree of cytopenias present at baseline, but these rarely led to dose reduction $(4.5 \%$ and $2.3 \%$ for thrombocytopenia and anemia, respectively) or discontinuation (3.8\% and $3.8 \%$, respectively). Among patients remaining on study, hemoglobin and platelet counts were stable through week 24 (Figure 4). While thrombocytopenia was observed more often on pacritinib, there was no excess of hemorrhagic events (pacritinib vs. BAT: grade $\geq 1$, $51.5 \%$ vs. $59.6 \%$; grade $3-4,13.6 \%$ vs. $10.5 \%$; fatal, $2 \%$ vs. $0 \%$ ). High-grade and fatal cardiac events were observed at similar rates on pacritinib and BAT (grade 3-4, 9.1\% vs. 14\%;

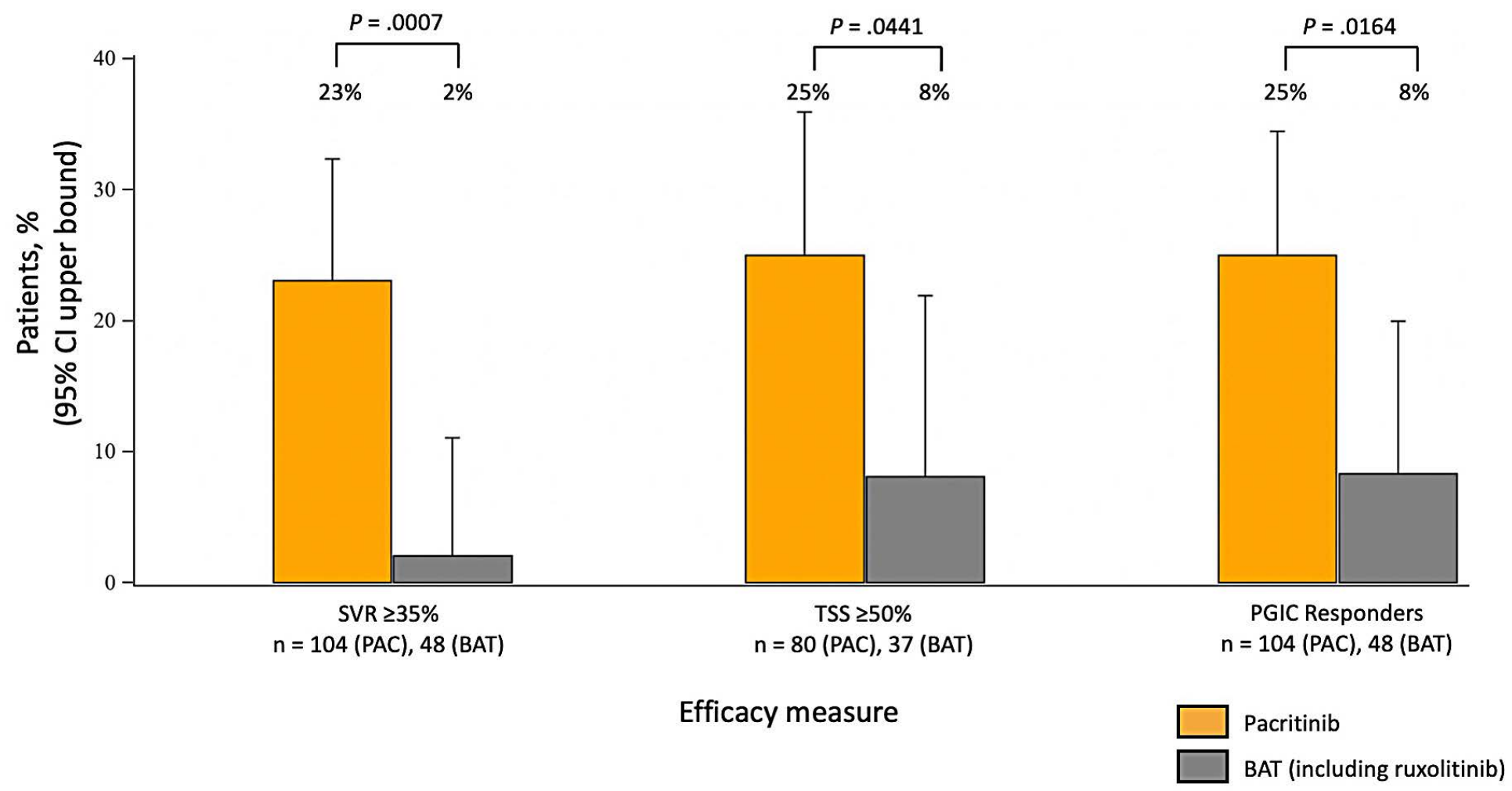

Figure 1. Efficacy of pacritinib versus best available therapy based on 24-week response rates in patients with severe thrombocytopenia. Graph depicts the percentage of patients achieving $\geq 35 \%$ spleen volume reduction (SVR), achieving $\geq 50 \%$ reduction in modified Total Symptom Score (TSS), and reporting symptoms as being "much" or "very much" improved based on Patient Global Impression of Change (PGIC) at week 24. Percentages are based on all patients randomized at least 22 weeks prior to the termination of the PERSIST studies (intention-to-treat [ITT] population). BAT: best available therapy; CI: confidence interval; PAC: pacritinib. 
A

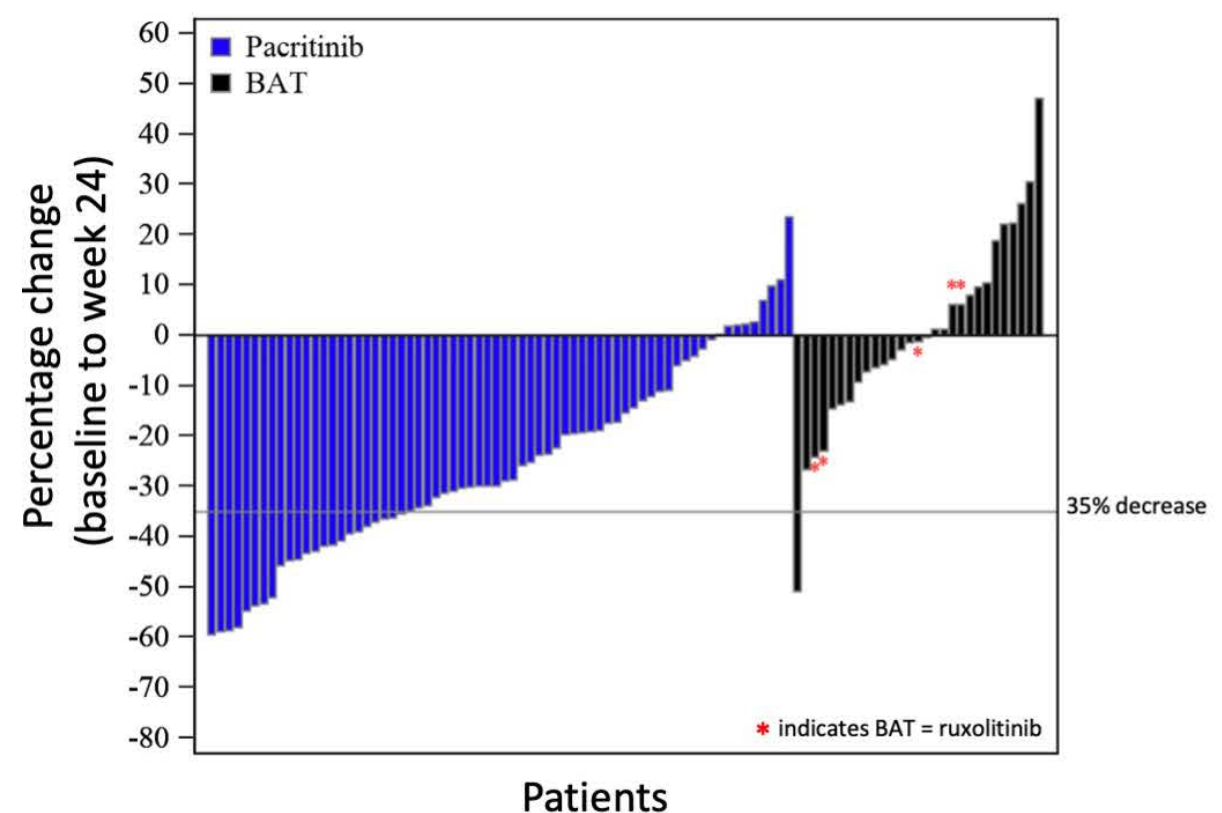

B

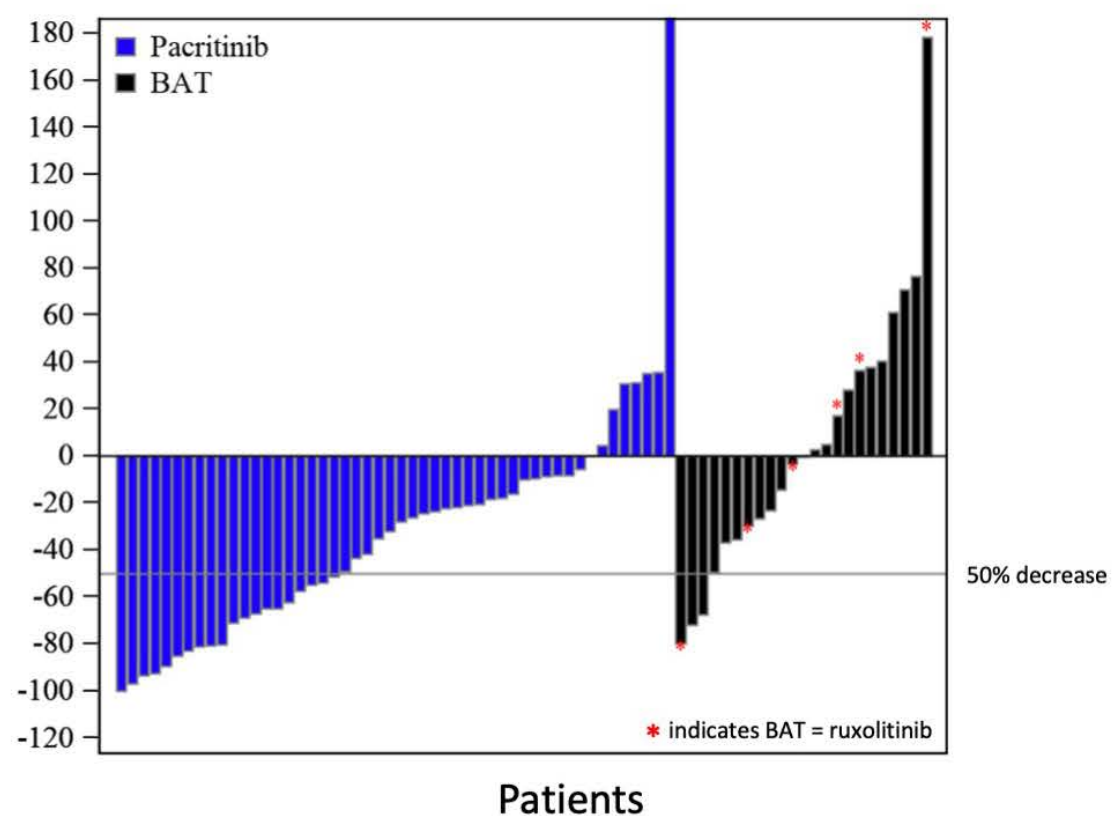

Figure 2. Waterfall plots of percentage change from baseline. (A) Change in spleen volume and (B) change in modified Total Symptom Score (TSS) at week 24 in patients with severe thrombocytopenia. Data are shown for evaluable patients treated with pacritinib (pooled dose groups) or best available therapy (BAT) (including ruxolitinib, indicated with red asterisks). Gray horizontal lines indicate responder threshold (35\% for spleen volume reduction [SVR], 50\% for TSS).

Table 2. Spleen volume response and modified Total Symptom Score response rates among patients randomized to pacritinib versus best available therapy by subgroup: prior exposure to a JAK2 inhibitor (including ruxolitinib) and myelofibrosis subtype (primary vs. secondary after a prior diagnosis of polycythemia vera or essential thrombocythemia)

\begin{tabular}{|c|c|c|c|}
\hline Response rate at week 24 & Pacritinib & BAT & P-value* \\
\hline \multicolumn{4}{|c|}{ Patients with $\geq 35 \%$ spleen volume reduction, $\%(n / N)$} \\
\hline $\begin{array}{l}\text { Prior JAK2 inhibitor exposure } \\
\text { Yes } \\
\text { No }\end{array}$ & $\begin{array}{l}17.9(5 / 28) \\
17.9(5 / 28)\end{array}$ & $\begin{array}{c}7.7(1 / 13) \\
0(0 / 35)\end{array}$ & 0.07 \\
\hline $\begin{array}{l}\text { MF diagnosis } \\
\text { Primary } \\
\text { Secondary }\end{array}$ & $\begin{array}{c}24.0(18 / 75) \\
20.7(6 / 29)\end{array}$ & $\begin{array}{c}3.1(1 / 32) \\
0(0 / 16)\end{array}$ & 0.52 \\
\hline \multicolumn{4}{|c|}{ Patients with $\geq 50 \%$ reduction in modified TSS, $\%(n / N)$} \\
\hline $\begin{array}{l}\text { Prior JAK2 inhibitors } \\
\text { Yes } \\
\text { No }\end{array}$ & $\begin{array}{c}17.9(5 / 28) \\
28.8(15 / 52)\end{array}$ & $\begin{array}{c}15.4(2 / 13) \\
4.2(1 / 24)\end{array}$ & 0.14 \\
\hline $\begin{array}{l}\text { MF diagnosis } \\
\text { Primary } \\
\text { Secondary }\end{array}$ & $\begin{array}{c}30.4(17 / 56) \\
12.0(3 / 25)\end{array}$ & $\begin{array}{c}12.5(3 / 24) \\
0(0 / 12)\end{array}$ & 0.43 \\
\hline \multicolumn{4}{|c|}{ Patients with "much" or "very much" improved PGIC scores, \% (n/N) } \\
\hline $\begin{array}{l}\text { Prior JAK2 inhibitors } \\
\text { Yes } \\
\text { No }\end{array}$ & $\begin{array}{c}14.3(4 / 28) \\
28.9(22 / 76)\end{array}$ & $\begin{array}{c}15.4(2 / 13) \\
5.7(2 / 35)\end{array}$ & 0.08 \\
\hline $\begin{array}{l}\text { MF diagnosis } \\
\text { Primary } \\
\text { Secondary }\end{array}$ & $\begin{array}{c}30.7(23 / 75) \\
10.3(3 / 29)\end{array}$ & $\begin{array}{c}12.5(4 / 32) \\
0(0 / 16)\end{array}$ & 0.45 \\
\hline
\end{tabular}

BAT: best available therapy; JAK2: Janus kinase 2; MF: myelofibrosis; PGIC: Patient Global Impression of Change; SVR: spleen volume reduction; TSS: Total Symptom Score. *Breslow and Day homogeneity test 
fatal, $3 \%$ vs. 1.8\%). Survival was similar between pacritiniband BAT-treated patients: hazard ratio (HR): 1.01 (95\% confidence interval [Cl]: 0.57-1.80).

\section{Discussion}

In this retrospective analysis, the pacritinib group was associated with improved SVR and symptom response compared to BAT in patients with MF and severe thrombocytopenia, a population with an unmet need for safe and effective therapies. Efficacy was observed irrespective of prior JAK2 inhibitor exposure or MF subtype (primary or secondary), although SVR and symptom response rates were numerically higher in patients who did not have prior JAK inhibitor exposure. Efficacy in this subgroup of patients with severe thrombocytopenia was similar to that observed in the PERSIST studies overall, including in patients with higher platelet counts. The patient population described in this analysis had advanced disease: in addition to severe thrombocytopenia (median platelet count $28 \times 10^{9} / \mathrm{L}$ ), about half of the patients had

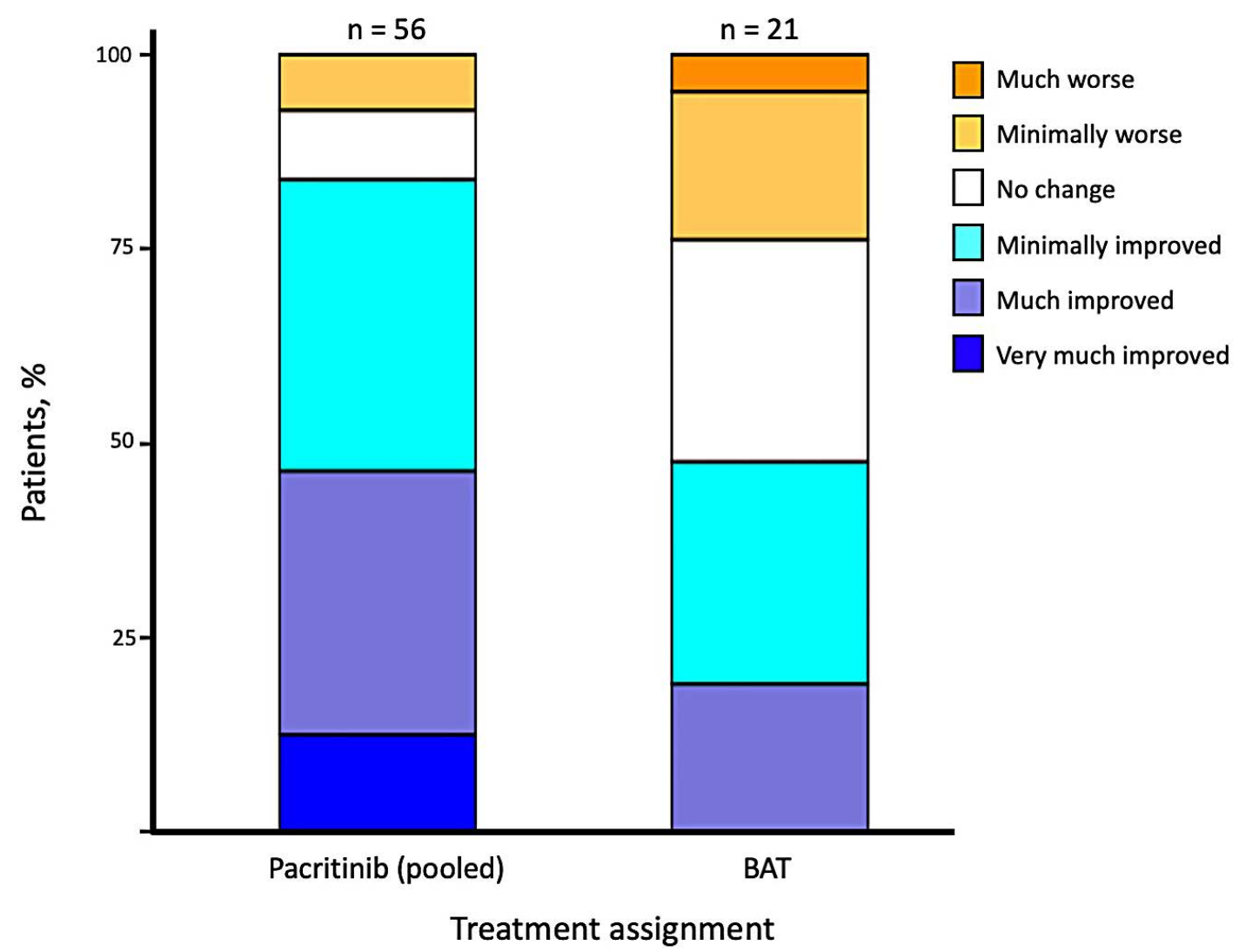

Figure 3. Self-reported symptoms in patients who completed the Patient Global Impression of Change at week 24 by treatment group. The percentage of evaluable patients with any improvement in disease symptoms was higher for patients randomized to pacritinib (84\% [47/56]) than for those randomized to best available therapy (BAT) (48\% [10/21]).

\section{Change in Hemoglobin Over Time}

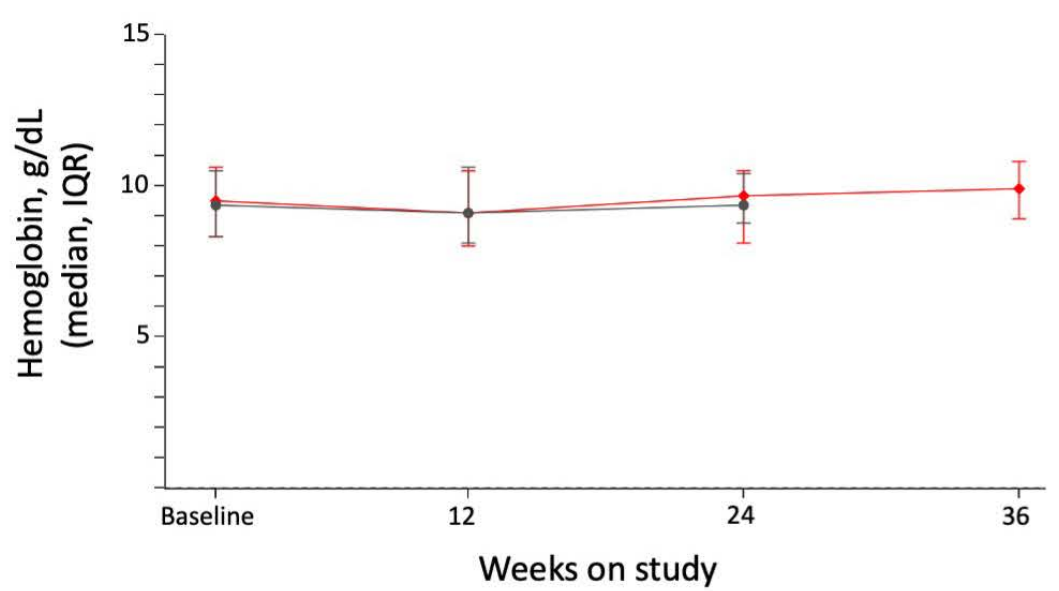

No. of patients

Pacritinib 132

BAT $\quad 56$
90

58
Change in Platelet Count Over Time

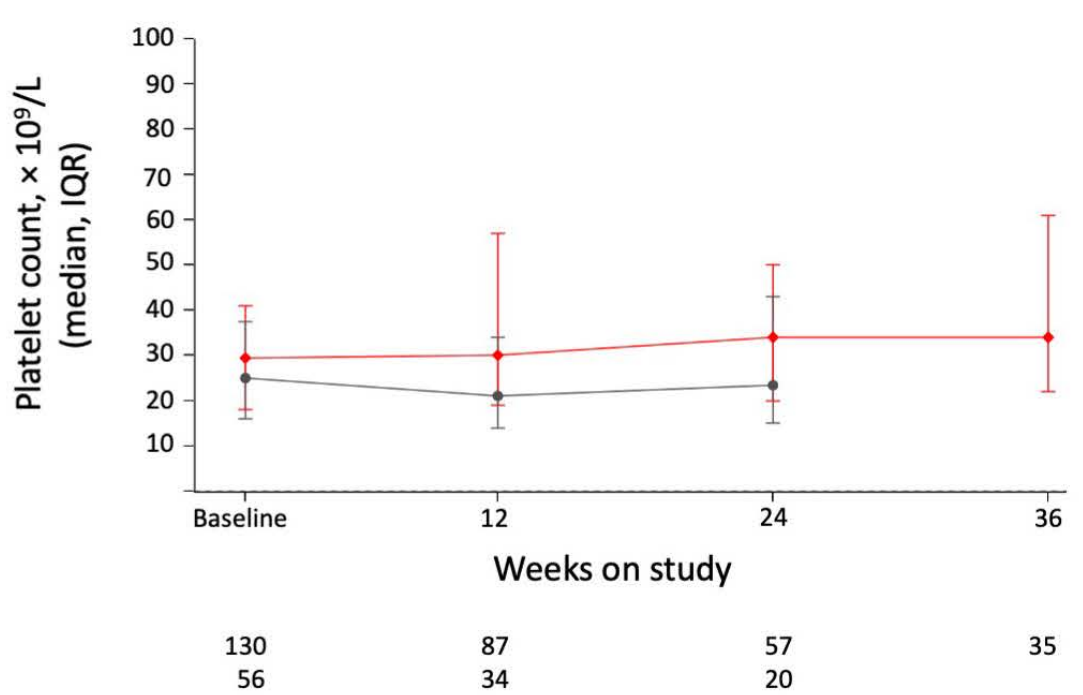

Figure 4. Median hemoglobin and platelet count over time through week 24. Among patients remaining on study, the median hemoglobin (A) and platelet count (B) remained stable over time in both pacritinib- and best available therapy (BAT)-treated patients. IQR: interquartile range. 
Table 3. Most common treatment-emergent adverse events ( $\geq 10 \%$ all grade or $\geq 3 \%$ grade 3 or 4 in either group) in all treated patients.*

\begin{tabular}{|c|c|c|c|c|}
\hline \multirow[t]{2}{*}{ TEAE, $\mathbf{N}(\%)$} & \multicolumn{2}{|c|}{ Pacritinib ( $N=132)$} & \multicolumn{2}{|c|}{ BAT $(N=57)$} \\
\hline & All grade & Grade 3-4 & All grade & Grade 3-4 \\
\hline Diarrhea & $80(60.6)$ & $7(5.3)$ & $9(15.8)$ & $9(15.8)$ \\
\hline Thrombocytopenia & $46(34.8)$ & $46(34.8)$ & $12(21.1)$ & $12(21.1)$ \\
\hline Anemia & $42(31.8)$ & $42(31.8)$ & $12(21.1)$ & $11(19.3)$ \\
\hline Nausea & $40(30.3)$ & $2(1.5)$ & $7(12.3$ & $1(1.8)$ \\
\hline Vomiting & $35(26.5)$ & $1(0.8)$ & $4(7.0)$ & $1(1.8)$ \\
\hline Epistaxis & $21(15.9)$ & $9(6.8)$ & $15(26.3)$ & $1(1.8)$ \\
\hline Peripheral edema & $21(15.9)$ & $2(1.5)$ & $13(22.8)$ & 0 \\
\hline Fatigue & $19(14.4)$ & $6(4.5)$ & $6(10.5)$ & $3(5.3)$ \\
\hline Dizziness & $18(13.6)$ & $2(1.5)$ & $2(3.5)$ & 0 \\
\hline Pyrexia & $17(12.9)$ & 0 & $6(10.5)$ & 0 \\
\hline Constipation & $17(12.9)$ & $1(0.8)$ & $4(7.0)$ & 0 \\
\hline Abdominal pain & $16(12.1)$ & $2(1.5)$ & $10(17.5)$ & $1(1.8)$ \\
\hline Dyspnea & $14(10.6)$ & $2(1.5)$ & $5(8.8)$ & $2(3.5)$ \\
\hline Pneumonia & $14(10.6)$ & $10(7.6)$ & $2(3.5)$ & $2(3.5)$ \\
\hline Decreased appetite & $14(10.6)$ & $3(2.3)$ & $4(7.0)$ & 0 \\
\hline Upper respiratory tract infection & $12(9.1)$ & 0 & $6(10.5)$ & $1(1.8)$ \\
\hline Contusion & $13(9.8)$ & 0 & $6(10.5)$ & 0 \\
\hline Cough & $10(7.6)$ & $1(0.8)$ & $7(12.3)$ & 0 \\
\hline Neutropenia & $8(6.1)$ & $7(5.3)$ & $4(7.0)$ & $4(7.0)$ \\
\hline Leukopenia & $7(5.3)$ & $5(3.8)$ & $2(3.5)$ & $2(3.5)$ \\
\hline Cardiac failure & $5(3.8)$ & $5(3.8)$ & $3(5.3)$ & $2(3.5)$ \\
\hline Atrial fibrillation & $2(1.5)$ & $1(0.8)$ & $4(7.0)$ & $2(3.5)$ \\
\hline General health deterioration & $4(3.0)$ & $4(3.0)$ & 0 & 0 \\
\hline Lower respiratory tract infection & $4(3.0)$ & 0 & $2(3.5)$ & $2(3.5)$ \\
\hline Sepsis & $4(3.0)$ & $2(1.5)$ & $3(5.3)$ & $2(3.5)$ \\
\hline Abdominal pain, upper & $7(5.3)$ & $1(0.8)$ & $3(5.3)$ & $2(3.5)$ \\
\hline
\end{tabular}

BAT: best available therapy; TEAE: treatment-emergent adverse event. *Events were counted regardless of whether they were considered related to study drug. Disease progression as an adverse event is not listed.

circulating blasts $\geq 1 \%$, and two-thirds had significant anemia (hemoglobin $<10 \mathrm{~g} / \mathrm{dL}$ ). These findings are consistent with previous reports describing the co-occurrence of these poor prognostic factors in patients with severe thrombocytopenia. ${ }^{2,3}$ Furthermore, the patients presented here had significant burden of disease, with spleen volumes consistent with those reported in patients with higher platelet counts. ${ }^{6,8,20}$ Interestingly, the majority of patients had hypo- or normocellular bone marrow, as opposed to the hypercellular marrow typically associated with myeloproliferative disease.
Pacritinib was tolerated at full doses in patients with severe thrombocytopenia, and the safety profile was consistent with that observed in the PERSIST studies overall, although rates of bleeding were higher in patients with severe thrombocytopenia regardless of whether they were treated with pacritinib or BAT. The most common adverse events were gastrointestinal, and these were predominantly low grade and manageable with anti-diarrheals. Despite severe thrombocytopenia at baseline, discontinuation due to myelosuppression was rare for patients on pacritinib. Furthermore, hemorrhagic events 
were observed at similar frequencies for pacritinib and BAT, suggesting that bleeding is more likely associated with disease-related thrombocytopenia ${ }^{21}$ and platelet dysfunction. ${ }^{22}$

In summary, pacritinib was shown to be more effective than BAT in reducing splenomegaly and symptom burden in patients with MF and severe thrombocytopenia. While this analysis is post hoc and retrospective, the results highlight the important role that pacritinib may play in the future therapeutic landscape for patients living with cytopenic MF. In the recent PAC203 phase II dose-finding study conducted in patients with advanced and heavily pretreated MF, including those with severe thrombocytopenia, the SVR response rate for patients with severe thrombocytopenia was $17 \%,{ }^{23}$ similar to that observed in this review (23\%); differences could be attributed to the enrollment of patients with prolonged duration of prior ruxolitinib exposure on PAC203 (median duration of exposure was 2.1 years). The randomized phase III PACIFICA study is currently under way in patients with MF and severe thrombocytopenia comparing pacritinib $200 \mathrm{mg}$ BID with physicians' choice of therapy. Results from PACIFICA should confirm whether pacritinib will be a new therapeutic option for patients with cytopenic MF.

\section{Disclosures}

SV reports consulting for CTI BioPharma. RM reports consulting for Novartis, Sierra Oncology, La Jolla Pharma, and AOP; and research support from Incyte, Gilead, CTI BioPharma, Celgene, AbbVie, and Genentech. MT reports serving on advisory boards for Novartis, BMS, and Constellation; and conducting clinical studies supported by Novartis, Constellation, and BMS. J-JK reports serving on advisory boards for Novartis, BMS/Celgene, AbbVie, Incyte, $A O P$ Orphan, and CTI BioPharma. CNH reports research funding from Novartis, Celgene, and Constellation; and institutional and speaker funding from Novartis, Celgene, CTI BioPharma, Gilead, Shire, Roche, Janssen, Promedior, Geron, Galacteo, and AOP. STO reports consulting/advisory boards for Disc Medicine, Blueprint Medicines, PharmaEssentia, Constellation, Geron, AbbVie, Sierra Oncology, Incyte, Kartos Therapeutics, CTI Biopharma, and Celgene/BMS. AMV reports serving on advisory boards for Novartis, Incyte, AbbVie, and Celgene/BMS; and lecture fees from Novartis, CTI BioPharma, Celgene/BMS, and AbbVie.
$R R$ reports consulting fees from Constellation, Incyte, Celgene/BMS, Promedior, CTI Biopharma, Jazz Pharmaceuticals, Blueprint, Stemline, Galecto, Pharmaessentia, Kartos Therapeutics, and AbbVie; and research funding from Incyte, Constellation, and Stemline. BLS reports serving on advisory committees for Celgene/BMS and Alexion; and research funding from Novartis. SAB, ARC, and KR-T are employees of CTI BioPharma, and ARC is a stockholder in CTI BioPharma, Inc. JOM reports research funding paid to his institution from Incyte, Roche, Forbius, CTI BioPharma, Merck, AbbVie, Novartis, Geron, Kartos Therapeutics, and PharmaEssentia; and serving in a consulting or advisory role for Celgene/BMS, Constellation, Incyte, Novartis, Roche, Sierra, CTI BioPharma, Genentech/Roche, Gilead Sciences, Promedior, PharmaEssentia, KartosTherapeutics, and AbbVie.

\section{Contributions}

$R M, M T, J-J K, C N H, S V$, and JOM contributed to the study design; $S B$ and KR-T collected the data; all authors performed data analysis and interpretation; and all authors participated in manuscript preparation and approval of the final version.

\section{Acknowledgments}

Editing assistance was provided by Maxine Skipp, of Twist Medical, LLC. This assistance was funded by TMAC Advisors.

\section{Data-sharing statement}

CTI is committed to enhancing appropriate transparency of research in a responsible manner and improving access to clinical trial information in order to inform medical decision-making, advance scientific discovery and accelerate development of new treatments to benefit patients. This includes data sharing, registration and results reporting, voluntary disclosures and lay/plain language summaries. Clinical trial results for PERSIST 2 (NCT02055781) and PAC203 (NCT04884191) have been previously published (Mascarenhas et al. JAMA Oncol. 2018;4(5):652-659 and Gerds et al. Blood Adv. 2020;4(22): 5825-5835) and have been or will be posted to ClinicalTrials.gov and other public registries as required. Inquiries regarding availability of data and clinical trial documentation will be considered on a case-by-case basis and should be directed to: Medinfo@ctibiopharma.com.)

\section{References}

1. Scotch AH, Kosiorek H, Scherber R, et al. Symptom burden profile in myelofibrosis patients with thrombocytopenia: Lessons and unmet needs. Leuk Res. 2017;63:34-40.

2. Hernandez-Boluda JC, Correa JG, Alvarez-Larran A, et al. Clinical characteristics, prognosis and treatment of myelofibrosis patients with severe thrombocytopenia. $\mathrm{Br} \mathrm{J}$ Haematol. 2018;181(3):397-400.

3. Masarova L, Alhuraiji A, Bose $P$, et al. Significance of thrombocytopenia in patients with primary and postessential thrombocythemia/polycythemia vera myelofibrosis. Eur $\mathrm{J}$ 
Haematol. 2018;100(3):257-263.

4. Jakafi (ruxolitinib) [package insert]. Wilmington, DE: Incyte; 2020.

5. Inrebic (fedratinib) [package insert]. Summit, NJ: Celgene Corporation; 2019.

6. Verstovsek S, Mesa RA, Gotlib J, et al. A double-blind, placebocontrolled trial of ruxolitinib for myelofibrosis. N Engl J Med. 2012;366(9):799-807.

7. Harrison CN, Vannucchi AM, Kiladjian JJ, et al. Long-term findings from COMFORT-II, a phase 3 study of ruxolitinib vs best available therapy for myelofibrosis. Leukemia. 2016;30(8):1701-1707.

8. Pardanani A, Harrison C, Cortes JE, et al. Safety and efficacy of fedratinib in patients with primary or secondary myelofibrosis: a randomized clinical trial. JAMA Oncol. 2015;1(5):643-651.

9. Harrison CN, Schaap N, Vannucchi AM, et al. Janus kinase-2 inhibitor fedratinib in patients with myelofibrosis previously treated with ruxolitinib (JAKARTA-2): a single-arm, open-label, non-randomised, phase 2, multicentre study. Lancet Haematol. 2017;4(7):e317-e324.

10. Center for Drug Evaluation and Research: Ruxolitinib Clinical Pharmacology and Biopharmaceutics Review (NDA 202192). https://www.accessdata.fda.gov/drugsatfda_docs/nda/2011/2021 920rig1s000clinPhar.

11. Kuykendall AT, Shah S, Talati C, et al. Between a rux and a hard place: evaluating salvage treatment and outcomes in myelofibrosis after ruxolitinib discontinuation. Ann Hematol. 2018;97(3):435-441.

12. Guglielmelli P, Kiladjian JJ, Vannucchi A, et al. The final analysis of EXPAND: a phase 1b, open-label, dose-finding study of ruxolitiminb in patients with myelofibrosis and low platelet count $\left(50 \times 10^{9} / \mathrm{L}\right.$ to $\left.<100 \times 10^{\%} / \mathrm{L}\right)$ at baseline. Blood. 2020;136(Suppl 1):S4-5.

13. Al-Ali HK, Griesshammer M, Foltz L, et al. Primary analysis of JUMP, a phase $3 b$, expanded-access study evaluating the safety and efficacy of ruxolitinib in patients with myelofibrosis, including those with low platelet counts. $\mathrm{Br} \mathrm{J}$ Haematol. 2020;189(5):888-903.

14. Masarova L, Mesa RA, Hernández-Boluda JC, Taylor JA. Severe thrombocytopenia in myelofibrosis is more prevalent than previously reported. Leuk Res. 2020;91:106338.

15. Singer JW, Al-Fayoumi S, Ma H, Komrokji RS, Mesa R, Verstovsek S. Comprehensive kinase profile of pacritinib, a nonmyelosuppressive Janus kinase 2 inhibitor. J Exp Pharmacol. 2016;8:11-19.

16. Mesa RA, Vannucchi AM, Mead A, et al. Pacritinib versus best available therapy for the treatment of myelofibrosis irrespective of baseline cytopenias (PERSIST-1): an international, randomised, phase 3 trial. Lancet Haematol. 2017;4(5):e225-e236.

17. Mascarenhas J, Hoffman R, Talpaz M, et al. Pacritinib vs best available therapy, including ruxolitinib, in patients with myelofibrosis: a randomized clinical trial. JAMA Oncol. 2018;4(5):652-659.

18. Verstovsek S, Odenike O, Singer JW, Granston T, Al-Fayoumi S, Deeg HJ. Phase $1 / 2$ study of pacritinib, a next generation JAK2/FLT3 inhibitor, in myelofibrosis or other myeloid malignancies. J Hematol Oncol. 2016;9(1):137.

19. Emanuel RM, Dueck AC, Geyer HL, et al. Myeloproliferative neoplasm (MPN) symptom assessment form total symptom score: prospective international assessment of an abbreviated symptom burden scoring system among patients with MPNs. J Clin Oncol. 2012;30(33):4098-4103.

20. Harrison C, Kiladjian JJ, Al-Ali HK, et al. JAK inhibition with ruxolitinib versus best available therapy for myelofibrosis. $\mathrm{N}$ Engl J Med. 2012;366(9):787-798.

21. Hasselbalch $\mathrm{H}$. Idiopathic myelofibrosis: a clinical study of 80 patients. Am J Hematol. 1990;34(4):291-300.

22. Leoni P, Rupoli S, Lai G, et al. Platelet abnormalities in idiopathic myelofibrosis: functional, biochemical and immunomorphological correlations. Haematologica. 1994;79(1):29-39.

23. Gerds AT, Savona MR, Scott BL, et al. Determining the recommended dose of pacritinib: results from the PAC203 dose-finding trial in advanced myelofibrosis. Blood Adv. 2020;4(22):5825-5835.

24. Gale RP, Barosi G, Barbui T, et al. What are RBC-transfusiondependence and -independence? Leuk Res. 2011;35(1):8-11. 